\title{
An elderly man with an irritable hip
}

\author{
M D Vahidassr, K Dynan, C J Foy, A P Passmore
}

A 79-year-old man was transferred for rehabilitation following transurethral resection of prostate (benign nodular hyperplasia). Rehabilitation progressed satisfactorily until the occurrence of some lower back pain. There was no history of trauma or systemic ill-health. Initial investigations showed an elevated C-reactive protein (CRP) $69 \mathrm{mg} / 1$ (normal <10); alkaline phosphatase 732 IU/1 (normal range 90-280) which then reduced to $280 \mathrm{IU} / \mathrm{l}$. Initial investigations, including a spine X-ray, were otherwise inconclusive. Simple analgesia offered good pain relief, he was mobile with a zimmer frame and was discharged home 10 weeks after admission.

He was re-admitted 3 weeks later with excruciating lower back pain radiating down the left leg, refractory to simple analgesia. Clinical examination revealed an alert man with a pyrexia of $37.6^{\circ} \mathrm{C}$. Examination of his back revealed no bony tenderness. There was symmetrical muscle wasting of both legs. Left hip flexion was painful (power grade 4/5) and straight leg raising was restricted to $45^{\circ}$. There was loss of sensation in all modalities affecting the lateral aspect of his left leg with absent knee jerk.

Investigations revealed haemoglobin $11.1 \mathrm{~g} / \mathrm{dl}$ (normocytic normochromic), alkaline phosphatase $983 \mathrm{IU} / 1, \gamma$-glutamyl transpeptidase $278 \mathrm{IU} / \mathrm{l}$ (5-60), calcium and phosphate normal, erythrocyte sedimentation rate (ESR) $115 \mathrm{~mm} / \mathrm{h}, \mathrm{CRP} 72 \mathrm{mg} / \mathrm{l}$, prostate-specific antigen level normal, mid-stream sample of urine normal; chest X-ray and abdominal ultrasound were unremarkable. Lumbar spine X-ray and isotope bone scan are shown in figures 1 and 2, respectively.

Belfast City Hospital, Belfast, Northern Ireland $M$ D Vahidassr K Dynan

\section{Craigavon Area}

Hospital, Craigavon, Northern Ireland C J Foy

Department of Geriatric Medicine, The Queen's University of Belfast, Belfast, Northern Ireland

A P Passmore

Correspondence to Dr MD Vahidassr, Department of Geriatric Medicine, Whitla Medical Building, 97

Lisburn Road, Belfast BT9 7BL

Accepted 10 March 1998

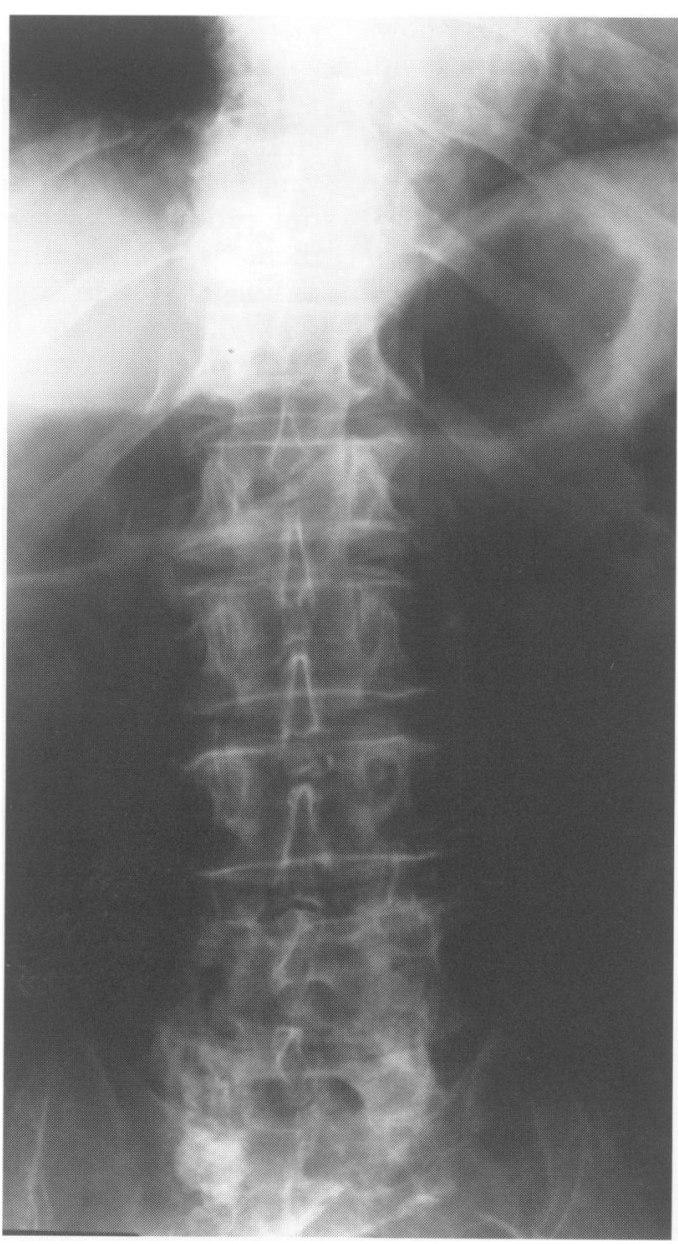

Figure 1 Lumbar spine X-ray

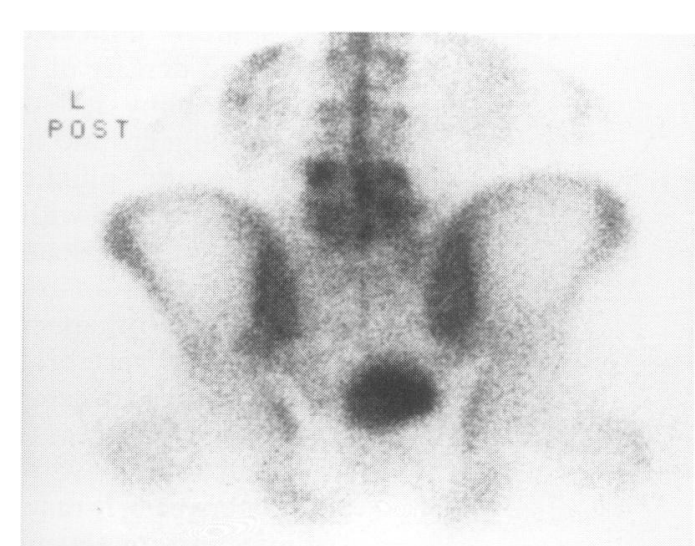

Figure 2 Isotope bone scan

\section{Questions}

1 What do the lumbar spine X-ray and isotope bone scan show?

2 What is the anatomical diagnosis?

3 What is the differential diagnosis?

4 What single diagnostic investigation is indicated? 


\begin{tabular}{|l|}
\hline Differential diagnosis \\
\hline Bony origin \\
— lumbar spondylosis \\
- primary bone neoplasm \\
- secondary bony metastasis \\
- vertebral osteomyelitis \\
- intervertebral disc protrusion \\
Neurological \\
— neuromas \\
\hline
\end{tabular}

\section{Answers}

QUESTION 1

The lumbar spine $\mathrm{X}$-ray and isotope bone scan show marked degenerative changes with lumbar lordosis and increased uptake at L4 and L5.

\section{QUESTION 2}

The anatomical diagnosis is a compressive lesion at lower lumbar root segments.

QUESTION 3

The differential diagnosis is shown in the box.

QUESTION 4

Computed tomography (CT) scan of the lumbar spine is indicated (figure 3 ). This shows increased density of bodies of L4 and L 5 with extensive bony destruction of L4. The soft tissue swelling around the vertebral body extends to involve the spinal canal with narrowing and the psoas muscle with calcification. CT-guided drainage of the psoas shadow and subsequent bacteriological analysis revealed the presence of acid-fast organisms. A final diagnosis of tuberculous vertebral osteomyelitis with secondary psoas abscess was made.

Iliopsoas abscess is relatively uncommon with an annual world-wide incidence of 12 cases per year. ${ }^{1}$ It may be primary with no focus of infection, or secondary, as a result of direct spread from adjacent structures. Primary abscess is becoming more prevalent ${ }^{12}$; this increase is related to intravenous drug abuse and HIV infection. The majority are due to

1 Gruenwald I, Abrahamson J, Cohen O. Psoas abscess: case report and review of the literature. 7 Urol 1992;147:1624-6. report and review of the literature. $f$ Prol $1992 ; 147: 1624-6$. ary psoas abscess. Presentation, microbiology, and treatary psoas abscess. Presentation, micr
ment. Arch Surg 1995;130:1309-13.

3 Ricci MA, Rose FB, Meyer KK. Pyogenic psoas abscess: world-wide variation in aetiology. World F Surg 1986;10: 834-43.

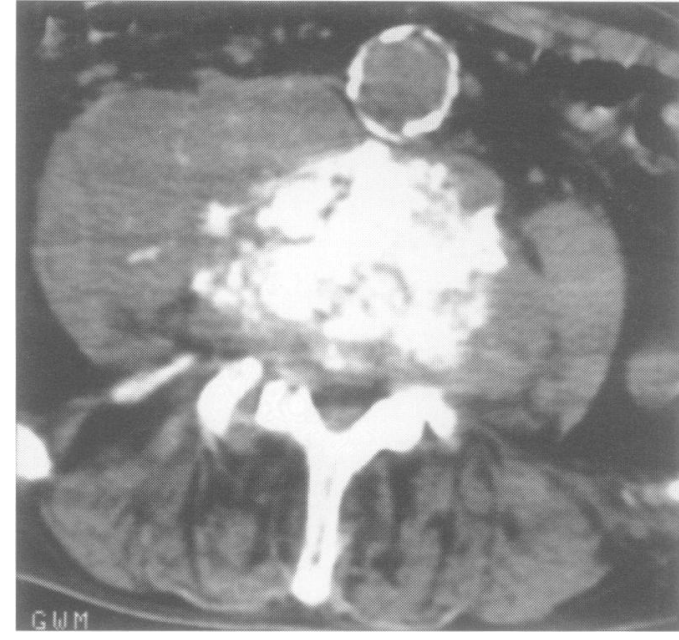

Figure 3 CT scan of lumbar spine

infections by Staphylococcus aureus. ${ }^{3}$ Enteric organisms secondary to gastrointestinal pathology account for $80 \%$ of secondary abscesses. ${ }^{23}$ The incidence of abscesses secondary to $\mathrm{Myco}$ bacterium tuberculosis has declined significantly. ${ }^{4}$ Signs of psoas muscle irritation include an irritable hip which is most comfortable when in a flexed position, positive iliopsoas sign, and limp on ambulation. ESR and CRP are elevated. CT scan is the investigation of choice with very high diagnostic rates. Characteristic calcification within the muscle is often seen in tuberculous psoas abscess. ${ }^{5}$ Involvement of adjacent vertebrae with frank destruction signals advanced disease because proteolytic enzymes are not produced by tuberculous bacilli. ${ }^{5}$ Treatment is with antituberculous therapy and drainage is often suggested. ${ }^{2}$ Although now a rare diagnosis, it is prudent to consider tuberculosis as a cause in older people and the immunosuppressed.

\section{Final diagnosis}

Tuberculous vertebral osteomyelitis with secondary psoas abscess.

Keywords: tuberculous vertebral osteomyelitis; psoas abscess

4 Procaccino JA, Lavery IC, Fazio VW, et al. Psoas abscess: dfficulties encountered. Dis Colon Rectum 1991;34:784-9.

5 Harrigan RA, Kauffman FH, Love MB. Tuberculous psoas abscess. $\mathcal{F}$ Emerg Med 1995;13:493-8. 